\title{
SUL GRADIENTE ADIABATICO DI TEMPERATURA NELL'INTERNO DELLA TERRA
}

\author{
Paolo Emilio Valle
}

1. - Il gradiente adiabatico di temperatura nell'interno della Terra ha una notevole importanza in molte questioni di carattere geofisico. Esso è espresso dalla nota relazione termodinamica

$$
\left(\frac{\partial T}{\partial p}\right)_{\mathrm{s}}=\frac{T \alpha}{\varrho c_{\mathrm{p}}}
$$

nella quale $T$ è la temperatura assoluta, $a$ la dilatazione termica, Q la densità, $c_{\mathrm{r}}$ il calore specifico a pressione costante ed $S$ l'entropia.

Se si ammette l'equilibrio idrostatico e si indica con $h$ la profondità e con $g$ l'accelerazione di gravità, risulta

$$
d p=\varrho g d h
$$

e la [1] può essere scritta nella forma

$$
\left(\frac{\partial T}{\partial h}\right)_{\mathrm{s}}=\frac{T \mathrm{ug}}{c_{\mathrm{p}}}
$$

o anche

$$
d \log T=\frac{\alpha g}{c_{\mathrm{p}}} d h
$$

Fino a qualche anno $\mathrm{fa}$, il rapporto $u / c_{\mathrm{p}}$ veniva dedotto da misure di laboratorio eseguite su varie rocce ignee. Nel mantello della Terra il suo valore medio era ritenuto pari a circa $1.510^{-12} \mathrm{~g}$ erg $^{-i}$.

Recentemente J. Verhoogen ( ${ }^{1}$ ) ha proposto un nuovo metodo per la valutazione del suddetto rapporto, allo scopo di eliminare l'inconveniente dell'estrapolazione dei valori ottenuti in laboratorio, alle alte pressioni esistenti nel mantello della Terra.

Il suo procedimento richiede però alcune semplificazioni, le quali appaiono alquanto arbitrarie. In questa nota viene indicato un metodo per il calcolo del rapporto $\alpha / c_{\mathrm{p}}$, basato sulla teoria di un solido ideale, nell'ipotesi che la temperatura assoluta sia un poco superiore alla temperatura di Debye relativa alle onde longitudinali. Si am- 
mette poi la reversibilità dei processi termodinamici, ciò che del resto è già implicito [1].

Viene effettuato il calcolo del rapporto $\alpha / c_{p}$ e l'integrazione della [3] per il mantello.

L'aumento di temperatura per compressione adiabalica nel nucleo, viene valutato soltanto nello strato $E$, mediante l'estensione del metodo sopra accennato.

2. - È noto (2-3-4) clie un solido, il quale, in scguito ad una dilatazione o compressione isotropa ed omogenea, assuma il volume $V$, reagisce alla pressione esterna $P$ con due tipi di pressioni interne: l'uno dovuto arli sforzi statici, l'altro all'agitazione termica. Mentre la valutazione del primo tipo di pressione interna ̀̀ piuttosto difficile e richiede una dettagliata conoscenza della struttura del solido, la valutazione del secondo tipo di pressione interna ̀̀ relativamente semplice.

Gli atomi di un solido non possono muoversi liberamente, ma oscillano intorno a determinate posizioni di equilibrio, facendo nascere onde elastiche completamente diffuse.

L'agritazione termica può essere associata a queste onde, la cui lunghezza però non può essere inferiore al doppio della distanza tra due particelle. Ne consegue che lo spettro delle frequenze i limitato superiormente.

Se si indicano con l'indice $l$ le grandezze che si riferiscono alle onde longitudinali e con l'indice $t$ le grandezze che si riferiscono alle onde trasversali, la frequenza massima è data da

$$
v_{\mathrm{m}}=v_{\mathrm{m}}\left(\frac{3 N}{4 \pi V}\right)^{\frac{1}{3}} \quad(\boldsymbol{m}=l, \boldsymbol{t})
$$

nella quale $v_{m}$ è la velocità, supposta indipendente dalla frequenza, ed $N$ il numero di atomi contenuti nel volume $V$, che nel secuito verrà assunto come volume specifico.

La pressione dovula all'agritazione termica, coincide con la pressione di radiazione dei due tipi di onde, e può scriversi ( $(2)$

$$
p_{\mathrm{m}}=\frac{U_{\mathrm{m}}}{V}\left(\frac{1}{3}-\frac{\partial \log v_{\mathrm{m}}}{\partial \log V}\right) \quad(m=l, t)
$$

$U_{\text {u }}$ rappresenta l'energia media di oscillazione.

L'equazione di stato di un solido ideale, il quale sia sottoposto soltanto a variazioni isotrope ed omogenee di volume, è quindi della forma

$$
p=f(V)+\Sigma_{m} p_{m} \quad(m=l, t, t)
$$


La pressione di radiazione delle onde trasversali deve essere contata due volte in relazione alle due possibili direzioni di vibrazione di queste onde.

Posto

$$
p_{\mathrm{i}}=\Sigma_{\mathrm{n}} p_{\mathrm{m}} \quad(m=l, t, t)
$$

si ottiene facilmente dalla [4]

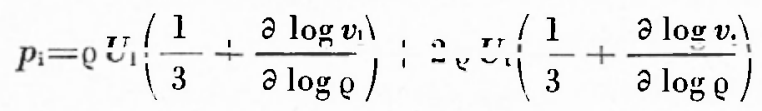

I.e energie medie di oscillazione $U_{1}$ ed $U_{1}$ sono pari all'energia complessiva di $N$ oscillatori, considerati come indipendenti, la cui frequenza non può superare $v_{1}$ e $v_{1}$ rispettivamente.

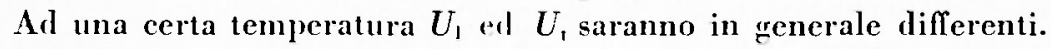
Si suppongra che la temperatura sia alquanto superiore alla temperatura di Delye relativa alle onde longitudinali, essa sarà superiore anche alla temperatura di Debye relativa alle onde trasversali, dato che queste ultime possegrgono una velocità minore delle prime. In questo caso è facile vedere ${\left({ }^{2-3-4}\right)}$ che l'energia media di oscillazione dei due tipi di onde, tende arl assumere il comune valore classico $N k T$, dove $k$ è la costante di Boltzmann, e quindi si potrà scrivere

$$
U_{1}=U_{\mathrm{t}}=U
$$

e l'energria media totale sarà pari a $3 U$, poiché l'enerria media delle onde trasversali deve essere contata due volte.

La pressione interna di radiazione assume allora la forma

$$
p_{\mathrm{i}}=\varrho U \frac{\partial \log \left(o v_{1} v_{1}^{2}\right)}{\partial \log \varrho}
$$

Si indichino ora con $x_{\mathrm{r}}$ e $x_{i}$ rispettivamente la compressibilità isoterma ed adiabatica, con $c_{v}$ il calore specifico a volume costante, da note relazioni di termodinamica, si ha

ossia

$$
\begin{gathered}
\left(\frac{\partial p}{\partial T}\right)_{\mathrm{v}}=\frac{a}{x_{\mathrm{r}}} \\
\frac{x_{\mathrm{r}}}{\boldsymbol{x}_{\mathrm{a}}}=\frac{c_{\mathrm{p}}}{c_{\mathrm{v}}}
\end{gathered}
$$

$$
\frac{\alpha}{c_{\mathrm{p}}}=\left(\frac{\partial p_{\mathrm{i}}}{\partial T T}\right)_{\mathrm{v}} \frac{\boldsymbol{x}_{\mathbf{s}}}{x_{\mathrm{v}}}
$$


nella quale

$$
c_{\mathrm{v}}=3\left(\frac{\partial U}{\partial T}\right)_{\mathrm{v}}
$$

Tenendo conto della $\left[6^{\prime}\right]$ e della [8] si ottiene dalla [7]

ricordando poi che

$$
\frac{\alpha}{c_{\mathrm{p}}}=\varrho x_{\mathrm{s}} \frac{\partial \log \left(\varrho v_{1} v_{\mathrm{t}}^{2}\right)^{1 / 3}}{\partial \log 0}
$$

$$
\varrho x_{\mathrm{s}}=\frac{1}{v_{\mathrm{1}}^{2}-\frac{4}{3} v_{\mathrm{t}}^{2}}
$$

risulta in definitiva

$$
\frac{\alpha}{c_{\mathrm{v}}}=\frac{1}{v_{1}^{\bar{z}}-\frac{4}{3} v_{\mathrm{i}}^{2}} \frac{\partial \log \left(\varrho v_{1} v_{1}^{2}\right)^{1 / 3}}{\partial \log 0}
$$

E questa una relazione che consente il caìcolo diel rapportort $/ c_{n}$, mediante la conoscenza della velocita delle onde longitudinali e trasversali e della densiti, indipendentemente dalla composizione chimica del solido. Pertanto questa relazione si presenta particolarmente adatta per il calcolo di $\alpha / c_{\mathrm{n}}$ nel mantello della Terra.

Il gradiente adiabatico si ottiene sostituendo la [11] nella [3] e si ha

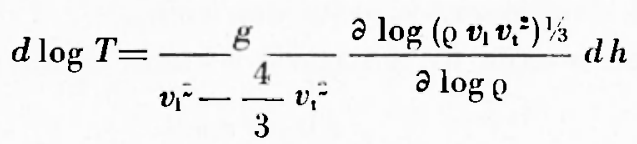

Si consideri infine uno strato di materiale omogeneo, nel quale la variazione della densita sia dovuta alla sola compressione adiabatica. Si avrà allora

$$
x_{\mathrm{s}} g=\frac{1}{\varrho}\left(\frac{\partial \varrho}{\partial h}\right)
$$

e quindi dalla [12], tenuto conto della [10], risulta

$$
\frac{T^{3}}{\varrho v_{1} v_{\mathrm{s}}}=\text { costanie }
$$

come si era già ottenuto in un precedente lavoro $\left({ }^{5}\right)$ per altra via. 
Questa equazione può essere applicata agli strati nei quali l'andamento della densità è stato calcolato supponendo l'equilibrio adialatico e una costituzione omogenea.

3. - La temperatura di Delye relativa alle onde longitudinali alla profondità di $33 \mathrm{~km}$ è presumibilmente alquanto inferiore a $1000^{\circ} \mathrm{K}$, mentre in prossimità del nucleo vale circa $2000^{\circ} \mathrm{K}$. Queste temperature non sembrano essere eccessivamente elevate e si può quindi ritenere che l'ipbtesi sotto la quale è stata dedotta la [11] sia verificata.

Per la valutazione del rapporto $\alpha / c_{1}$, occorre conoscere la velocita di propagazione delle onde longitudinali e trasversali in funzione della densità. Ora la conoscenza dei dati sismici, della massa, della densití media e del momento di inerzia, non è sufficiente a determinare univocamente l'andamento della densità nell'interno della Terra.

Pertanto il calcolo di tale andamento è stato eseguito sotto varie ipotesi ed è stata calcolata più di una distriluzuione della densità.

In hase ai valori della velocità delle onde sismiche calcolati da H. Jeffreys $\left(^{6-\tau}\right), K$. E. Bullen $\left({ }^{8}\right)$, allo scopo di eliminare l'indeterminazione del prollema, considera due casi: i) che la densità vari in modo continuo attraverso tutto il nucleo centrale, e cioè nelle regioni $E, F$ e $G$, ii) che la densità al centro della Terra sia più grande di $10 \mathrm{gcm}^{-3}$ che nel caso i). Le due ipotesi conducono ad una distribuzione

Tabella I

\begin{tabular}{|c|c|c|c|c|c|c|}
\hline $\begin{array}{l}h \\
\mathrm{~km}\end{array}$ & $\lim ^{\prime \prime} \sec ^{-1}$ & $\mathrm{kn}^{1} \sec ^{-}$ & $\mathrm{g} \mathrm{cm}^{0}$ & $\begin{array}{l}p 10^{-12} \\
\text { dine } \mathrm{cm}^{-2}\end{array}$ & $\begin{array}{ll}g & g \\
\operatorname{cm} \operatorname{ser}^{-z}\end{array}$ & $0^{1 / 3}\left(0 v_{1} v_{t}^{2}\right)^{1 / 3}$ \\
\hline 33 & 7,75 & 4,35 & 3,32 & 0,009 & 985 & 11.7359 \\
\hline 100 & 7,95 & 4,45 & 3,38 & 0,031 & 989 & 12.1611 \\
\hline 200 & 8,26 &,+ 60 & 3,47 & 0,065 & 992 & 12.8150 \\
\hline 300 & 8,58 &,+ 76 & 3,55 & 0,100 & 995 & 13,4809 \\
\hline 413 & 8,97 &,+ 96 & 3,64 & $0,1+1$ & 998 & $11,299.1$ \\
\hline 500 & 9,56 & 5,28 & 3,89 & 0,173 & 1000 & 15,9174 \\
\hline 600 & 10,25 & 5,66 & $.1,13$ & 0,213 & 1001 & 17,7590 \\
\hline 800 & 11.00 & 6,13 & 4,19 & 0,300 & 999 & 20,2738 \\
\hline 1000 & 11,42 & 6,36 & $\begin{array}{r}4,68 \\
\end{array}$ & 0,392 & 995 & 21.6283 \\
\hline 1200 & 11,71 & 6,50 & $\begin{array}{r}4,80 \\
\end{array}$ & 0,19 & 991 & 22,5055 \\
\hline 1400 & 11,99 & 6,62 & 4,91 & 0.58 & 988 & 23,3112 \\
\hline 1600 & 12,26 & 6,73 & 5,03 & 0,68 & 986 & 24,1296 \\
\hline 1800 & 12,53 & 6.83 & 5,13 & 0,78 & 985 & $2.4,8699$ \\
\hline 2000 & 12,79 & 6,93 & 5,24 & 0,88 & 986 & $25,6+48$ \\
\hline 2200 & 13,03 & 7,02 & 5,34 & 0,99 & 990 & 26.3572 \\
\hline 2.400 & 13,27 & 7,12 & 5,41 & 1,09 & 998 & 27,1024 \\
\hline 2600 & 13,50 & 7,21 & 5,54 & 1,20 & 1009 & 27,8232 \\
\hline 2800 & 13,64 & 7,30 & 5,63 & 1,32 & 1026 & 28,4550 \\
\hline 2898 & 13,64 & 7,30 & 5,68 & 1,37 & 1037 & 28,6232 \\
\hline
\end{tabular}


della densità lievemente diversa nel mantello, mentre nel nuclco si ha un divario più accentuato. Successivamente W. II. Ramsey (") e lo stesso Bullen $\left({ }^{10}\right.$ ) hanno studiato nuove distribuzioni della densita, basate su altre ipotesi.

D'altra parte anche l'andamento della velocità delle onde sismiche nell'interno della Terra ¿̀ stato ricalcolato da B. Gutenberg (11), partendo da nuovi dati sperimentali, e i suoi risultati difieriscono in modo abbastanza sensibile dai valori precedentemente calcolati da Jeffreys.

L'Autore della presente ricerca si è servito delle velocità calcolate da Jeffreys e, per quanto riguarda il mantello, delle densità calcolate da Bullen in un lavoro precelente alla formulazione delle ipotesi i) e ii) $\left.{ }^{12}\right)$. I valori delle densità usate sono intermedi fra quelli delle

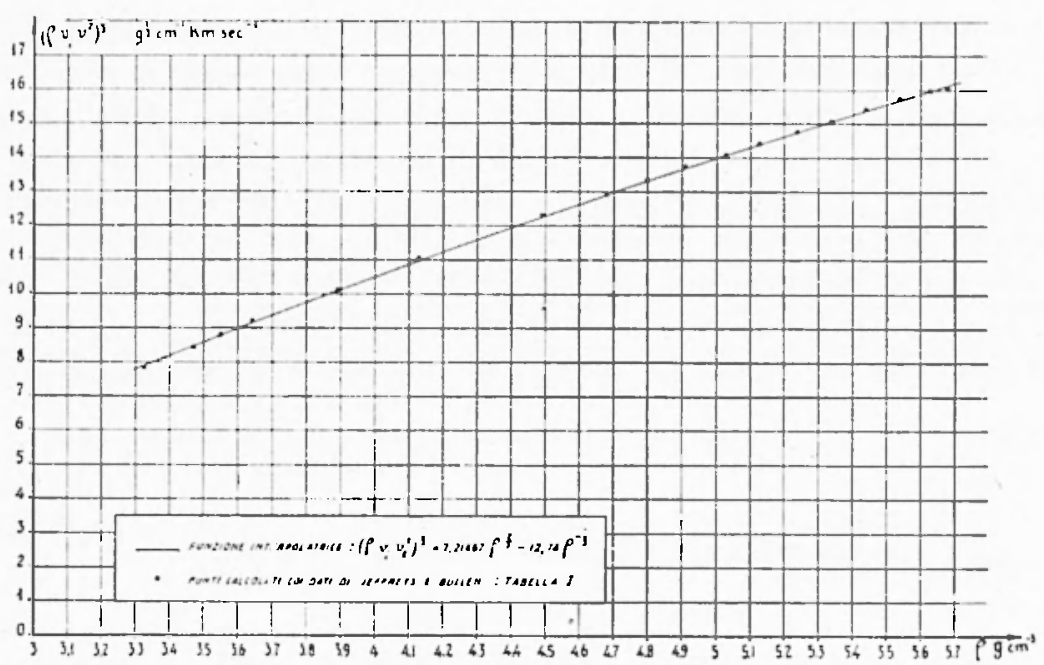

Fig. 1

due ipotesi e ne differiscono soltanto per qualche unità percentuale. Per quanto riguarda il nucleo sono stati considerati separatamente $i$ due casi sopraccennati.

Dal lavoro di Bullen sono stati tolti altresì i valori della gravita e della pressione, $i$ quali peraltro sono stati accuratamente calcolati anche da G. Boaga $\left({ }^{13-14}\right)$, che ne ha inoltre fornito le espressioni analitiche in funzione della profondita. I dati relativi al mantello sono contenuti nella tabella $I$ e i dati che si riferiscono al nucleo nella tabella IV.

4. - Nella valutazione del rapporto $\alpha / c_{\mathfrak{p}}$, l'operazione più delicata 
è costituita dal calcolo della derivata che compare a secondo membro della [11]

Si è notato che se si moltiplica per $\varrho^{1 / 3}$ l'espressione $\left(\varrho v_{1} v_{t}\right)^{1 / 3}$ si ottiene una funzione lineare nella $\varrho$. Pertanto si può scrivere

$$
Q^{1 / 3}\left(\varrho v_{1} v_{t}^{2}\right)^{1 / 3}=a+b \varrho
$$

Eccettuato il valore della funzione in prossimità del nucleo, l'espressione a secondo membro della precedente relazione rappresenta molto bene i valori del primo membro, calcolati con i dati della tabella $I$. Mediante il metodo dei minimi quadrati è stato calcolato il valore delle costanti $a$ e $b$, e si è ottenuto

$$
a=-12,14000 ; b=7,21467
$$

e quindi la [14] si può scrivere

$$
\left(0 v_{1} v_{\mathrm{t}}\right)^{2 / 3}=7,21467 \mathrm{Q}^{2 / 3}-12,14 \mathrm{Q}^{-1 / 3}
$$

Gli scarti $\&$, tra i valori calcolati mediante la [15] e i valori calcolati

\begin{tabular}{|c|c|c|c|}
\hline e & $\varepsilon$ & $\varrho$ & $\varepsilon$ \\
\hline 3,32 & $-0,0768$ & 4,80 & $+0,0151$ \\
\hline 3,38 & $-0,0815$ & 1,91 & +0.0272 \\
\hline 3,17 & $-0,0799$ & 5,03 & $-0,0202$ \\
\hline 3,55 & $+0,0088$ & 5,13 & -0.0014 \\
\hline 3.64 & $\begin{array}{r}+0,1780 \\
\end{array}$ & 5,24 & $-0,0201$ \\
\hline 3,89 & $-0,0077$ & 5,34 & $-0,0291$ \\
\hline 4,13 & $+0,1024$ & 5,44 & $-0,005.4$ \\
\hline 4,49 & $+0,0199$ & 5,54 & -0.0061 \\
\hline .7 .68 & +0.0036 & 5,63 & $-0,0236$ \\
\hline
\end{tabular}
dai dati della tabella I, sono contenuti nella tabella II.

TabelLa II

Lo scarto più elevato si trova in corrispondenza di $a=4,64$, cioè alla profondità di $413 \mathrm{~km}$, proprio in corrispondenza della discontinuità del sccondo ordine. Tale scarto non è però tanto elevato da introdurre seri errori nel calcolo del rapporto $\alpha / c_{\mathrm{p}}$.

La forma della [15] non sembra essere del tutto fortuita, almeno per quanto riguarda il primo termine a secondo membro. Da essa infatti si ricava

$$
\left(v_{1} v_{1}^{2}\right)^{1 / 3}=7,21467 \mathrm{e}^{1 / 3}-12,14 \mathrm{e}^{-2 / 3}
$$


Se si pensasse ora di estrapolare anmentando la densità fino a valori per i quali il secondo termine a secondo membro della [16] diventi trascurabile di fronte al primo, tenuto conto che la velocità delle onde

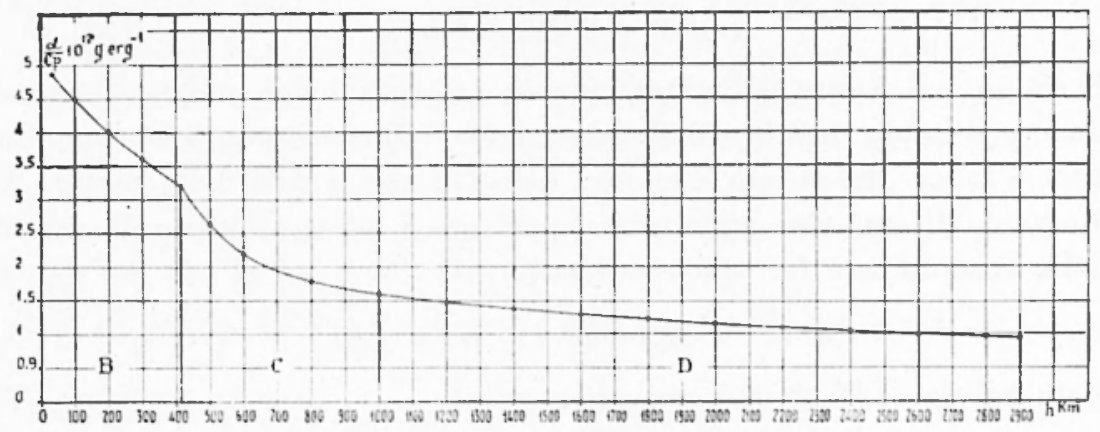

Fig. 2

trasversali sta in un rapporto press'a poco costante con la velocità delle onde longitudinali, si otterrebbe

$$
v_{1}=\operatorname{cost} \varrho^{1 / 3}
$$

Ora ad altissime densità corrispondono altissime pressioni, alle quali è lecito supporre cbe gli elettroni degli atomi si comportino quasi come un gas di Fermi $\left(^{15}\right)$.

Se questo punto di vista è griusto, la [17] deve avere la stessa forma dell'equazione che fornisce la velocità delle onde longitudinali in un gas di elettroni. Effettivamente, tenuto conto che per un gas di Fermi, a temperature non altissime, vale l'equazione di stato

$$
p=\operatorname{cost}^{5 / 3}
$$

si ottiene

$$
v_{1}=\operatorname{cost}_{e}^{1 / 3}
$$

che coincide formalmente con la [17]

Ulteriori considerazioni su questo argomento uscirebbero dai limiti del presente lavoro. 
TABELLA III

\begin{tabular}{|c|c|c|c|}
\hline $\begin{array}{c}\mathrm{l} \mathbf{l} \\
\mathrm{km}\end{array}$ & $\frac{\partial \log \left(o v_{1} v_{t}^{2}\right)^{1 / 3}}{\partial \log 0}$ & $\frac{\alpha}{c_{\mathrm{p}}} 10^{12} \mathrm{~g} \mathrm{erg}^{-1}$ & $\frac{T}{T_{83}}$ \\
\hline 33 & $1,69.4$ & 4.86 & 1 \\
\hline 100 & 1,658 & $+1,50$ & - \\
\hline 200 & 1,608 & 4,02 & $1,07_{\mathrm{u}}$ \\
\hline 300 & 1,568 & 3,61 & -- \\
\hline 413 & 1,526 & 3,20 & $1,16_{t}$ \\
\hline 500 & 1,429 & $2,63_{5}$ & - \\
\hline 600 & 1,354 & 2,17 & 1,21 。 \\
\hline 800 & 1,266 & $1,78_{5}$ & - \\
\hline 1000 & 1,228 & 1,60 & $1,31_{1}$ \\
\hline 1200 & $1,206_{5}$ & 1,49 & \\
\hline 1400 & 1,188 & 1,39 & $1.39_{1}$ \\
\hline 1600 & 1,169 & 1,30 & 一 \\
\hline 1800 & 1,155 & $1,2 \%$ & $1,46_{1}$ \\
\hline 2000 & 1,140 & 1.145 & 一 \\
\hline 2200 & 1,127 & 1,08 & 1,53 \\
\hline 2400 & $1.114_{5}$ & 1,03 & - \\
\hline 2600 & 1,103 & 0,98 & 1,59 。 \\
\hline 2800 & 1.093 & 0,95 & $1,62_{8}$ \\
\hline 2898 & $(1,088)$ & $(0,95)$ & $(1,64)$ \\
\hline
\end{tabular}

La seconda colonna della tabella III contiene $i$ valori di $\partial \log$ $\left(\varrho v_{1} v_{\mathrm{r}}^{2}\right)^{1 / 3} / \partial \log \varrho$ e la terza colonna il rapporto $\alpha / c_{\mathrm{p}}$, il cui andamento in funzione della profonditì è mostrato nella fig. 2. Nella fig. 3 è riportato l'andamento del rapporto $\alpha / c_{\mathrm{p}}$ con la pressione, ma è bene ricordare che la temperatura nell'interno della Terra non è costante. $\mathrm{Si}$ può tuttavia ammettere che alle alte pressioni esistenti nel man-

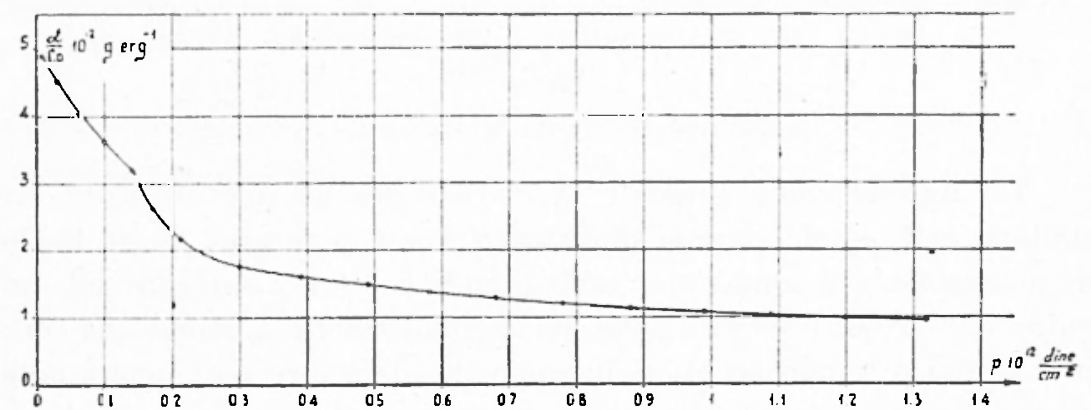

Fig. 3

tello, in prima approssimazione, l'influenza della temperatura sul rapporto $\alpha / c_{1}$ sia trascurabile, rispetto alla influenza della pressione.

Sotto questa ipotesi è stato calcolato il rapporto $T / T_{33}$, dove $T_{33}$ indica la temperatura a $33 \mathrm{~km}$, e i risultati sono contenuti nella ta- 
bella III. Da essi si rede che il gradiente adiabatico diminuisce al crescere della profondità. Se per es. si pone $T_{33}=1000^{\circ} \mathrm{K}$, si ottengono $i$ seguenti valori medi nei diversi strati.

S T R A T O

B

C

$\mathrm{D}$
GRADIENTE MIEDIO

gradi $\mathrm{km}^{-1}$

0,42

$0,25_{5}$

$0,17_{5}$

5. - A rigore, la teoria precedente non potrebbe essere applicata al nucleo, dato che esso, almeno per quanto riguarda lo strato $E$, si presenta come liquido.

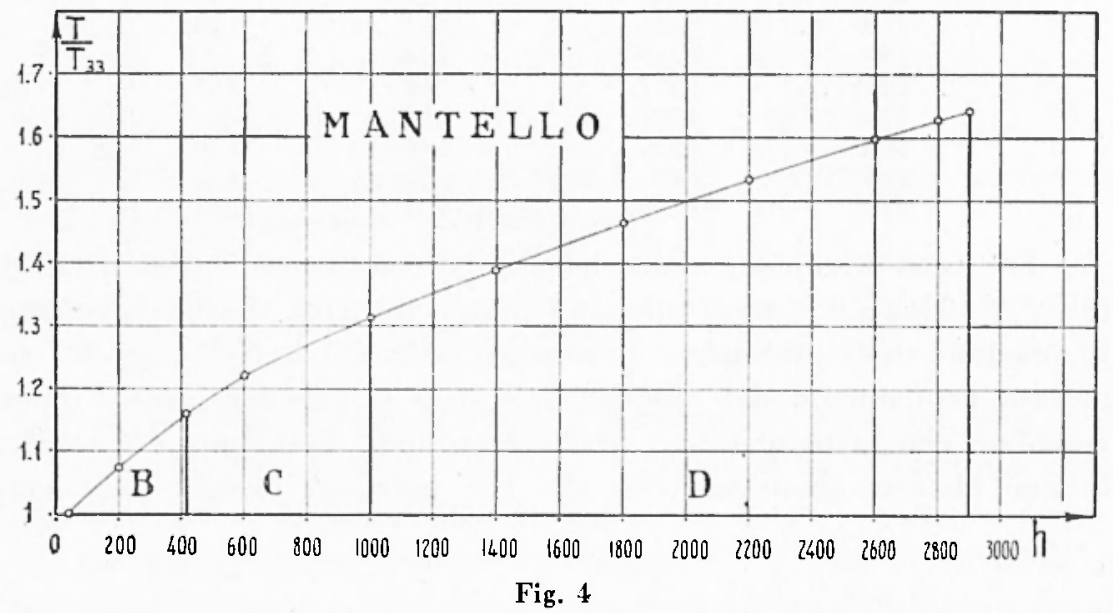

Un liquido come è noto $\left({ }^{2-4}\right)$, è costituito da una struttura cristallina, nella quale vi sono molti posti vuoti o, meglio, molte buche di potenziale. Gli atomi non sono quindi del tutto obbligati ad eseguire oscillazioni intorno a posizioni di equilibrio, ma hanno una certa probabilità di muoversi quasi liberamente dalla loro posizione attuale ad un'altra adiacente e momentaneamente vuota. Ne consegue che la pressione interna di agitazione termica non coincide esattanente con la pressione di radiazione delle onde longitudinali, le sole possibili in un liquido, né l'energia interna sarà pari all'energia di oscillazione media di queste onde. Peraltro sotto le fortissime pressioni :esistenti nel nucleo, è da presumere che un liquido conservi una struttura molto 
più vicina a quella del solido da cui proviene, di quanto non accada alle ordinarie pressioni di laboratorio.

In altre parole $\grave{e}$ da presumere che la percentuale delle buche di potenziale non sia troppo elevata.

Non sembra quindi del tutto arbitrario applicare la precedente tcoria anche al nucleo, con la condizione $v_{\mathrm{t}}=0$. Procedendo in modo analogo a quello indicato per lo stato solido, si ottiene facilmente la

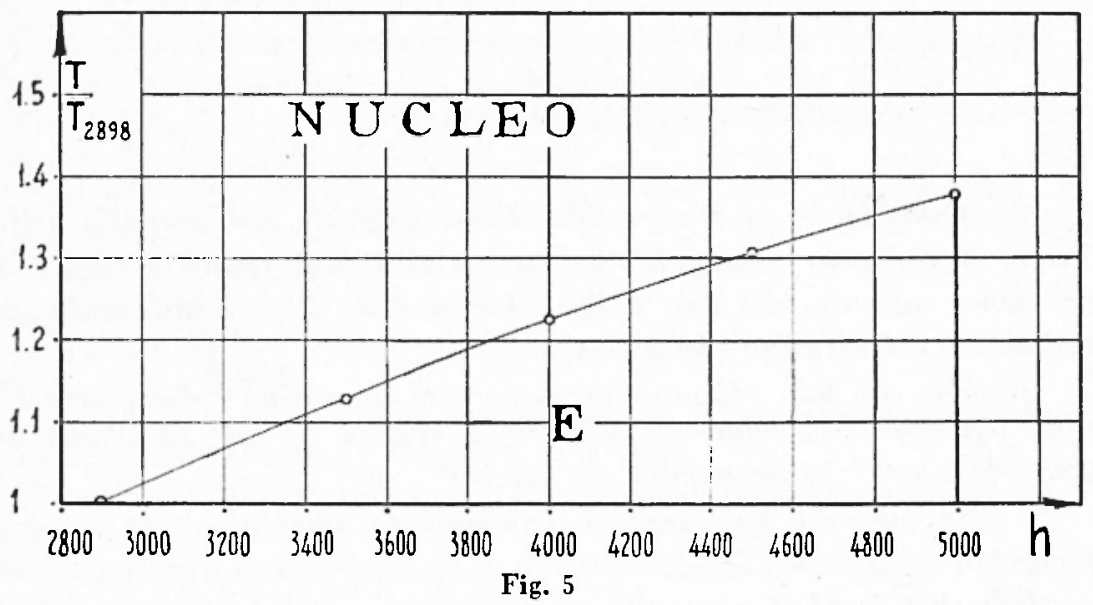

relazione che lega la densitá, la temperatura e la velocità delle onde longitudinali in una trasformazione adiabatica reversibile.

Si ha con qualche passaggio

$$
\frac{T^{3}}{\varrho v_{1}^{3}}=\text { costant } \mathrm{e}
$$

e non è necessaria la condizione che la temperatura sia superiore alla temperatura di Debye.

\section{TABELLA IV}

\begin{tabular}{|c|c|c|c|c|c|}
\hline $\begin{array}{c}h \\
\mathrm{~km}\end{array}$ & $\underset{\mathrm{g} \mathrm{cm}^{-3}}{\mathrm{(i)}}$ & $\underset{\mathrm{g}}{0} \mathrm{~cm}^{-3}$ & $\mathrm{~km} \sec ^{-1}$ & $\frac{T}{T_{2095}} \quad$ (i) & $\frac{\mathbf{T}}{\Gamma_{2393}}$ (ii) \\
\hline $\begin{array}{l}2898 \\
3500 \\
4000 \\
4500 \\
4982\end{array}$ & $\begin{array}{r}9,7 \\
10,5 \\
11,1 \\
11,6 \\
11,9\end{array}$ & $\begin{array}{r}9,1 \\
9,8 \\
10,3 \\
10,8 \\
11,1\end{array}$ & $\begin{array}{r}8,10 \\
8,9 \\
9,51 \\
9,97 \\
10,44\end{array}$ & $\begin{array}{c}1 \\
1,12_{8} \\
1,22_{8} \\
1,30_{\mathrm{o}} \\
1.38_{0}\end{array}$ & $\begin{array}{c}1 \\
1,12 。 \\
1,22 ، \\
1,30_{3} \\
1,37_{7}\end{array}$ \\
\hline
\end{tabular}


L'andamento della densití nello strato $E$ del nucleo, è stato calcolato da Bullen nell'ipotesi che in tutto lo strato il materiale sia omogeneo e l'equilibrio adiabatico. Pertanto l'equazione [18] è stata applicata a questo strato e i risultati sono contenuti nella tabella IV. Essi differiscono molto poco nei due casi i) e ii).

La fig. 5 mostra l'andamento del rapporto $T / T_{9 s 98}$ nel caso i).

Roma - Istituto Nazionale di Geofisica - Gennaio 1952.

\section{RIASSUNTO}

Mediante la teoria di un solido ideale si calcola, nel mantello della Terra, il rapporto tra la dilatazione termica e il calore specifico a pressione costante, dai dati della velocità delle onde sismiche longitudinali e trasversali e della densità.

Risulta che tale rapporto decresce con l'aumentare della profondità, passando dal valore di $4,8610^{-12} \mathrm{~g}$ erg $^{-1}$ a $3.3 \mathrm{~km}$, al valore di $0,9510^{-12} \mathrm{~g} \mathrm{erg}^{-1}$ in prossimità del nucleo.

Viene calcolato nel mantello l'andamento adiabatico con la profondità del rapporto $\mathrm{T} / \mathrm{T}_{33}$, dove $\mathrm{T}_{3: 3}$ è la temperatura a $33 \mathrm{hm}$, e se ne deducono $i$ valori approssimati del gradiente.

L'analogo rapporto $\mathrm{T} \mathrm{T}_{208}$ nello strato $\mathrm{E}$ del nucleo, viene valutato con l'estensione della teoria.

\section{$S L M M A R Y$}

On the basis of the theory of an ideal isotropic solid body, and starding from seismic data, the ratio of the coefficient of the thermal expansion to the specific heat at constant pressure in the Earth mantle, has been calculated.

It appears that such a ratio decreases by increasing of the depth, going from $4.8610^{-12} \mathrm{~g} \mathrm{erg}^{1}$ at the depth of $33 \mathrm{Km}$ to $0,9510^{12} \mathrm{~g}^{\mathrm{erg}}{ }^{1}$ near the bottom of the mantle.

The adiabatic behaviour of the ratio $\mathrm{T} / \mathrm{T}: 3, \mathrm{~T}_{: 3}$ means the temperature at $33 \mathrm{Km}$ of depth) by varying of the depth, has been calculated and the approximate values of the gradient have been deduced.

Through the extention of that theory, the similar ratio $\mathrm{T} / \mathrm{T}_{2898}$, referring to the E region of the core, has been estimated. 


\section{BIBLIOGRAFIA}

(1) Venhoogen J.: The Adiabatic Gradient in the Mantle. Trans. Amer. Geophys. Union $32,41-43$ (1951).

(-) Brillouin L.: Tenseurs en Mécanique et en Elasicite. Masson, Paris (1946).

(3) Bullovis L.: Wave Propagation in Periodic Structures. Mc Graw-Hill, New York (1946).

(4) Slater J. C.: Introduction to Chemical Physics. Me Graw-Hill, New York (1939).

(i) Valle P. E.: Sull'aumento di temperatura nel Mantello della Terra per compressione adiabatica. Ann. Geof., IV, 475-478 (1951).

(i) Jeffners H.: The Times of $P, S$ and SKS, and the Velocities of $P$ and $S$. M.N.R.A.S. Geophys. Suppl. IV, 498.533 (1939).

(1) Jefruses II.: The Times of the Core Waves (second paper). M.N.R.A.S. Geophys. Suppl. IV, 591-615 (1939).

(8) Bullev K. E.: Introduction of the Teory of Seismology. Cambridge, University Press (19:17).

(9) Ramsey W. H.: On the Nature of the Eartl's Core. M.N.R.A.S. Geophys. Suppl. V, 410-426 (1949).

(10) Bullen K. E.: An Earth Model Based on a Compressibility-Pressure Hypotesis. M.N.R.A.S. Geophys. Suppl. VI, 50.59 (1950).

(11) Gutengeng B.: On the Layer of relatively low Wave velocity at a Depth of about 80 hilometers. Bull. Seism. Soc. Am. 38, 121-148 (1948).

(10) Bullen K. E.: The Troblem of the Earth's Density Variation. Bull. Seism. Soc. Am. 30, 235-250 (19.10).

(13) Bosga G.: Sopra l'ipotesi di Bullen sulla variazione discontinua della densità nellinterno della Terra e sulla conseguente variazione della gravità. Pontificia Ass. Scient. V, n. 2 (1941).

(14) Boaga G.: Sulla variazione della pressione nellinterno della Terra con riferimento a variazione discontinua della densitio. Accademia d'Italia, Serie VII, III, 619.625 (19.12).

(15) Ransey W. H.: On the Compressibility of the Earth. M.N.R.A.S. Geophys. Suppl. VI, $42-49$ (1950). 